\title{
Mycobacterium avium SUBSP. paratuberculosis EN BOVINOS LECHEROS DE LA ZONA DE LIMA DETECTADO MEDIANTE TRES TÉCNICAS DIAGNÓSTICAS
}

\author{
Mycobacterium aVium SUbSp. PaRatuberculosis in DAIRy CatTle in the Lima \\ Area Detected through Three Diagnostic Techniques
}

José Bustamante V. 1,2, José Aguilar O. ${ }^{3}$, Martín Ortiz M. ${ }^{4}$, José Bustamante L. ${ }^{5}$

\section{Resumen}

\begin{abstract}
El presente estudio tuvo como objetivo evidenciar la presencia de Mycobacterium avium subsp. paratuberculosis (Map), en el ganado bovino lechero del departamento de Lima, Perú. Se evaluaron 60 vacas procedentes de tres establos, mediante la prueba tuberculínica intradérmica y dos kits comerciales de ELISA específicos contra Mycobacterium avium subsp. paratuberculosis. Resultaron positivos 22 animales (36.7\%) a una de las tres técnicas diagnósticas ( 8 a una de las pruebas de ELISA y 18 a la prueba intradérmica). Se tomó muestras de heces a los 22 animales positivos y se cultivaron en el medio de Herrold con yema de huevo, resultando cinco muestras positivas al aislamiento de Map. Asimismo, se realizó el PCR a partir del cultivo, encontrándose dos muestras positivas. Se encontró una concordancia ligera (0.15, prueba Kappa) entre las técnicas que evalúan la respuesta inmune celular y humoral y se estableció que estas pruebas no son reemplazables (5.55>3.84, prueba de Mc Nemar). Los resultados permitieron evidenciar la presencia de Map en ganado bovino lechero de Lima, Perú.
\end{abstract}

Palabras clave: paratuberculosis, enfermedad de Johne, Mycobacterium avium subsp. paratuberculosis, bacteria ácido alcohol resistente

\section{Abstract}

The objective of the study was to determine the presence of Mycobacterium avium subsp. paratuberculosis (Map) in dairy cattle in Lima, Peru. The Intradermal Tuberculin Test was applied to 60 cows from three herds and serum samples were tested using two commercial ELISA kits for antibodies against Map. Twenty-two cows (36.7\%) were positive to at least to one of these diagnostic techniques ( 8 to one of the ELISA tests and 18 to the intradermal test). Feacal samples from the 22 positive animals were cultured on Herrold's egg-yolk medium (HEYM) yielding five Map positive samples. PCR was used in the

\footnotetext{
${ }^{1}$ Facultad de Veterinaria y Zootecnia, ${ }^{3}$ Facultad de Ciencias y Filosofía, Universidad Peruana Cayetano Heredia, Lima.

${ }^{2}$ E-mail: joseabv27@hotmail.com

${ }^{4}$ Centro de Diagnóstico Sanidad Animal, SENASA, Lima

${ }^{5}$ Facultad de Medicina Veterinaria, Universidad Nacional Mayor de San Marcos, Lima
} 
isolated strains and Map DNA was confirmed in two cows. A slight concordance (0.15, Kappa test) was found between techniques evaluating cellular and humoral immune response, establishing that these tests are not interchangeable (5.55> 3.84, Mc Nemar test). The results confirm the presence of Map in dairy cattle in Lima, Peru.

Key words: paratuberculosis, Johne's disease, Mycobacterium avium subsp. paratuberculosis, acid-fast bacteria

\section{INTRODUCCIÓN}

El Mycobacterium avium subsp. paratuberculosis (Map), causa la enfermedad de Johne o paratuberculosis en el ganado vacuno, y es considerada por la Organización Mundial de Sanidad Animal (OIE) como una enfermedad de declaración obligatoria. Es una enfermedad entérica granulomatosa, progresiva y crónica que afecta a bovinos lecheros ocasionando grandes pérdidas económicas (Harris y Barletta, 2001; Motiwala et al., 2004). Algunos modelos de regresión estiman pérdidas que van desde US\$ 40 a 227 por vaca con sintomatología clínica (Harris y Barletta, 2001); asimismo, se calcula que causa US\$220 millones de pérdida por año en la industria lechera de los Estados Unidos (Waters et al., 2003; Coussens, 2004; Stabel et al., 2004; Huntley et al., 2005; Schuff, 2005).

Otra razón de la importancia de la enfermedad de Johne se basa en la posibilidad de que el Map sea el agente causal de la enfermedad de Crohn en el humano (HermonTaylor y Bull, 2002; Feller et al., 2007). Ambas enfermedades se han incrementado en los países industrializados (Schuff, 2005); sin embargo el papel de Map en la etiología de la enfermedad de Crohn queda por definirse (Feller et al., 2007). Bovinos con la enfermedad de Johne eliminan Map por la leche y es posible que la temperatura de la pasteurización por cortos periodos no sea efectiva para eliminar a la bacteria. Es posible, también, que la cepa clínica de Map pudiera ser más termotolerante que Mycobacterium bovis o Coxiella buernetii, microorganismos estándares de la pasteurización (Harris y Barletta, 2001). Esto podría condicionar la supervivencia del patógeno y afectar la salud del humano, causando un serio problema a la industria lechera (Koo et al., 2004; McKenna et al., 2006).

El diagnóstico presuntivo de la enfermedad puede realizarse en base a los síntomas clínicos y los hallazgos a la necropsia, pero requiere siempre la confirmación del laboratorio (Zúñiga, 1999; OIE, 2005). No es posible utilizar un sólo método para el diagnóstico, de allí la importancia del conocimiento de la respuesta inmune contra las micobacterias, a fin de diseñar la estrategia de aplicación de las diversas técnicas diagnósticas (SENASA, 2000). Los métodos de diagnóstico más usados son de tres tipos (Wren, 1998): detección de la inmunidad celular contra el patógeno, detección de la inmunidad humoral contra el patógeno y detección del patógeno.

Las pruebas para la detección de la inmunidad celular son las técnicas de elección en las etapas iniciales de la enfermedad, detectando animales con sintomatología subclínica (SENASA, 2000; De Juan Ferré, 2005). Dentro de estas, se tiene pruebas in vivo (tuberculínica intradérmica) e in vitro (gamma-interferón, transformación linfocitaria, inhibición de la migración leucocitaria, expresión del receptor de la Interleuquina 2) (De Juan Ferré, 2005).

Las pruebas para la detección de la inmunidad humoral detectan animales en estados clínicos de la infección o en estados finales de la enfermedad (SENASA, 2000; 
Stewart et al., 2006); pero son incapaces de identificar animales en una etapa temprana de la infección (De Juan Ferré, 2005). En este grupo se encuentran las pruebas de ELISA, inmunodifusión en gel de agar (AGID) y fijación de complemento (FC) (Collins et al., 2006).

Entre las pruebas para detectar Map, está el cultivo (Prueba de Oro) y el PCR, que son usadas como pruebas confirmatorias. La detección fecal de Map en animales infectados es la única metodología que permite detectar estados clínicos y subclínicos de la enfermedad en una manera confiable (Stabel y Bannantine, 2005); sin embargo, se tiene que considerar la eliminación intermitente de Map en heces hasta la última etapa de la infección. El cultivo microbiológico tiene, además, como desventaja el tiempo en que demora el crecimiento bacteriano (Jungersen et al., 2002).

El Perú era considerando como país libre de la enfermedad de Johne y, por lo tanto, no se tomaban las medidas de control respectivas; sin embargo, se sospechaba de su presencia en base a animales enfermos con signos clínicos compatibles con la enfermedad y por la presencia de animales seropositivos a la técnica de ELISA. A partir del 2008, el OIE registró oficialmente la presencia de la enfermedad de Johne en el Perú (OIE, 2009), aunque sin precisar el método diagnóstico reportado. El propósito del estudio fue evaluar la presencia de Map en el Perú.

\section{Materiales y Métodos}

Se trabajó en tres establos lecheros ubicados en las provincias de Huaura, Cañete y Lima, del departamento de Lima, Perú. Los establos (A, B y C) tenían historia de animales con signos clínicos compatibles con la enfermedad de Johne y contaban con una población de 566, 123 y 100 vacas, respectivamente. Previo al inicio al estudio, varios animales con signos clínicos compatibles con la enfermedad de Johne fueron beneficiados, incluyendo uno del establo $\mathrm{C}$ con diagnóstico histopatológico de inflamación granulomatosa crónica.

El tamaño de muestra calculado mediante la fórmula de prevalencia límite (Thrusfield, 1990) fue de 60 animales. El número de muestras por establo se calculó mediante la fórmula de muestras por estrato (Pagano y Gauvreau, 2001), resultando 43, 9 y 8 animales para los establos A, B y C. Se seleccionaron animales mayores a dos años de edad en forma aleatoria. Ninguno de estos animales presentaba signos clínicos de la enfermedad de Johne.

Se realizó la prueba tuberculínica intradérmica con el antígeno estandarizado PPD aviar, en una concentración de 1912 UI contenidas en $0.5 \mathrm{mg} / \mathrm{ml}$ y una potencia biológica de $76.5 \%$. El antígeno se preparó en el Laboratorio de Bacteriología del Centro de Diagnóstico de Sanidad Animal del SENASA, Perú. Se inyectó $0.1 \mathrm{ml}$ del inóculo en el tercio medio del cuello del bovino. El grosor de la piel se midió previo a la prueba y 72 horas posteriores a la inyección. Un incremento mayor a $3 \mathrm{~mm}$ fue considerado positivo.

Se tomaron muestras de sangre de la vena caudal en tubos al vacío sin anticoagulante. El suero resultante se obtuvo por centrifugación y se analizó mediante dos pruebas de ELISA. La primera fue con el HerdCheck M. paratuberculosis ELISA, de IDEXX Laboratories (Kit A) y se llevó a cabo en el Laboratorio de Microbiología e Inmunología de la Facultad de Veterinaria y Zootecnia de la Universidad Peruana Cayetano Heredia, Lima; y la segunda fue con el ELISA Paratuberculosis del Institut Pourquier, Montpellier, Francia (Kit B) y se hizo en el Laboratorio de Bacteriología del Centro de Diagnóstico de Sanidad Animal del Servicio Nacional de Sanidad AgrariaSENASA, Lima. La presencia o ausencia de anticuerpos para Map en cada muestra fue determinada por la proporción muestras/po- 
sitivas $(\mathrm{S} / \mathrm{P})$ siguiendo las recomendaciones señaladas en los manuales de cada kit. Se consideró como muestras positivas con el Kit $A$ a aquellas con un $\mathrm{S} / \mathrm{P}$ superior a $0.25 \mathrm{y}$ para el Kit B con un S/P superior o igual a 0.70 .

Se colectó una muestra de heces, vía rectal, a los animales positivos en al menos una de las tres pruebas. Para descontaminar las muestras, se colocó $1 \mathrm{~g}$ de heces en un tubo de vidrio que contenía $40 \mathrm{ml}$ de agua destilada estéril y la mezcla se agitó durante 30 minutos a temperatura ambiente; luego, 5 $\mathrm{ml}$ del sobrenadante se vació a un tubo con $35 \mathrm{ml}$ de solución de cloruro de hexadecilpiridinio al $0.75 \%$, se invirtió el tubo repetidas veces y se dejó a temperatura ambiente sin mover durante 24 horas (SENASA, 2000). A partir de allí, $0.1 \mathrm{ml}$ del sedimento se sembró en cuatro tubos con medio de Herrold, tres con micobactina y uno sin micobactina, y se cultivaron en forma horizontal, a $37^{\circ} \mathrm{C}$, en condiciones de aerobiosis, por una semana. Luego se les colocó en posición vertical, donde los tubos en donde sin crecimiento bacteriano fueron observados hasta por 6 meses.

La identificación de las bacterias se basó en:

- Caracterización de las colonias sospechosas: pequeñas, de aproximadamente $1 \mathrm{~mm}$ de diámetro, translúcidas, incoloras, hemisféricas y de superficie lisa y brillante.

- Ácido-alcohol-resistencia de las bacterias que forman la colonia sospechosa y su presentación en acúmulos.

- El tiempo de crecimiento superior al de otras micobacterias no tuberculosas.

- Ausencia de crecimiento en el tubo sin micobactina.

Las colonias de bacterias resultantes de analizaron por PCR para evaluar la amplificación de la secuencia de inserción IS900 presente en Map (Collins et al., 1993). Las muestras amplificadas se revelaron en un gel de agarosa al $1 \%$ teñido con bromuro de etidio al $5 \%$ y las bandas fueron observadas en un trasluminador UV. El producto amplificado fue de $280 \mathrm{pb}$. El PCR y el cultivo de las muestras se llevaron a cabo en el SENASA.

Se determinó la frecuencia de resultados positivos a Map en las pruebas de ELISA y PPDaviar. La concordancia de los resultados se evaluó con la prueba estadística de Kappa, en base al siguiente criterio (Gonzáles y Falcón, 1999): Concordancia ligera (0 $0.20)$, regular $(0.21-0.40)$ moderada $(0.41$ - 0.60), sustancial $(0.61-0.80)$ y perfecta (0.81 - 1.00). La prueba de Mc Nemar determinó si las técnicas diagnósticas son mutuamente reemplazables entre ellas para el diagnóstico de esta enfermedad. La significancia aceptada fue $<0.05$. Asimismo, la presencia de al menos un animal positivo permitiría detectar la presencia de la infección en un nivel de al menos la prevalencia límite fijada para el presente estudio (5\%).

Esta investigación tuvo la aprobación del Comité Institucional de Ética para el uso de Animales - CIEA, de la Universidad Peruana Cayetano Heredia, con código de inscripción 53146. Además, se obtuvo la carta de aceptación de los tres establos.

\section{Resultados}

Veintidós de las 60 vacas muestreadas (36.7\%) resultaron positivas, al menos, a una de las pruebas inmunológicas usadas. En 14 de estas (63.6\%), la respuesta inmune fue de tipo celular (PPD aviar), en 4 (18.2\%) fue de tipo humoral y en 4 (18.2\%) fue tanto de de tipo celular como humoral (Cuadro 1). Asimismo, se aislaron e identificaron colonias características a Map en $5(27.7 \%)$ de los 18 animales positivos a la prueba tuberculínica intradérmica, y se pudo confirmar el Map por PCR en 2 (11.1\%). Además, se aisló e identificó colonias características a Map en uno de los ocho animales positivos a la prueba de ELISA, el cual se reconfirmó por PCR (Cuadro 1). En total, se logró confirmar la identidad del agente biológico por PCR en dos animales. 
Cuadro 1. Asociación entre respuesta celular y humoral contra Map y la detección de Mycobacterium avium subsp. paratuberculosis en heces de vacas

\begin{tabular}{|c|c|c|c|c|c|}
\hline \multirow{2}{*}{ Establo $^{1}$} & \multirow{2}{*}{$\begin{array}{l}\text { Respuesta } \\
\text { celular }^{2}\end{array}$} & \multicolumn{2}{|c|}{ Respuesta humoral ${ }^{3}$} & \multirow{2}{*}{ Cultivo } & \multirow{2}{*}{ PCR } \\
\hline & & Kit A & Kit B & & \\
\hline A & Pos & Pos & Pos & $\mathrm{Neg}$ & $\mathrm{Neg}$ \\
\hline A & Pos & Neg & Neg & Pos & Pos \\
\hline A & Pos & Neg & Neg & $\mathrm{Neg}$ & $\mathrm{Neg}$ \\
\hline $\mathrm{A}$ & Pos & $\mathrm{Neg}$ & $\mathrm{Neg}$ & Neg & $\mathrm{Neg}$ \\
\hline A & Pos & $\mathrm{Neg}$ & Neg & Pos & $\mathrm{Neg}$ \\
\hline $\mathrm{A}$ & Neg & Pos & Pos & $\mathrm{Neg}$ & $\mathrm{Neg}$ \\
\hline A & Pos & $\mathrm{Neg}$ & Neg & $\mathrm{Neg}$ & $\mathrm{Neg}$ \\
\hline $\mathrm{A}$ & Pos & Pos & Pos & Pos & Pos \\
\hline A & Neg & Pos & Neg & Neg & $\mathrm{Neg}$ \\
\hline A & Pos & $\mathrm{Neg}$ & Neg & $\mathrm{Neg}$ & $\mathrm{Neg}$ \\
\hline $\mathrm{A}$ & Pos & Pos & $\mathrm{Neg}$ & $\mathrm{Neg}$ & $\mathrm{Neg}$ \\
\hline A & Neg & $\mathrm{Neg}$ & Pos & Neg & $\mathrm{Neg}$ \\
\hline $\mathrm{A}$ & Pos & $\mathrm{Neg}$ & Neg & Pos & $\mathrm{Neg}$ \\
\hline $\mathrm{A}$ & Pos & Neg & $\mathrm{Neg}$ & Neg & $\mathrm{Neg}$ \\
\hline $\mathrm{A}$ & Pos & Pos & Pos & $\mathrm{Neg}$ & $\mathrm{Neg}$ \\
\hline $\mathrm{A}$ & Pos & $\mathrm{Neg}$ & $\mathrm{Neg}$ & $\mathrm{Neg}$ & $\mathrm{Neg}$ \\
\hline A & $\mathrm{Neg}$ & Pos & $\mathrm{Neg}$ & $\mathrm{Neg}$ & $\mathrm{Neg}$ \\
\hline A & Pos & $\mathrm{Neg}$ & $\mathrm{Neg}$ & $\mathrm{Neg}$ & $\mathrm{Neg}$ \\
\hline B & Pos & $\mathrm{Neg}$ & Neg & Pos & $\mathrm{Neg}$ \\
\hline $\mathrm{C}$ & Pos & $\mathrm{Neg}$ & $\mathrm{Neg}$ & $\mathrm{Neg}$ & $\mathrm{Neg}$ \\
\hline $\mathrm{C}$ & Pos & $\mathrm{Neg}$ & Neg & $\mathrm{Neg}$ & $\mathrm{Neg}$ \\
\hline $\mathrm{C}$ & Pos & $\mathrm{Neg}$ & $\mathrm{Neg}$ & $\mathrm{Neg}$ & $\mathrm{Neg}$ \\
\hline
\end{tabular}

${ }^{1} 22$ muestras positivas en por lo menos una prueba diagnóstica

${ }^{2}$ Prueba tuberculínica intradérmica

${ }^{3}$ Kit A: HerdCheck M. paratuberculosis ELISA (IDEXX Laboratories); Kit B: ELISA Paratuberculosis (Institut Pourquier, Montpellier, Francia)

En el establo A se encontraron cuatro bovinos positivos al cultivo y dos positivos a la prueba de PCR. No había animales con signos clínicos compatibles a la enfermedad de Johne al inicio del estudio, pero la consistencia de las heces de algunos animales era menor a lo normal.

La prueba estadística Kappa (0.15) determinó una concordancia ligera entre las pruebas que evalúan la respuesta inmune celular y la respuesta inmune humoral; y la prueba de Mc Nemar (5.55>3.84) señaló que no son pruebas reemplazables.

La concordancia entre la prueba tuberculínica y el Kit A (Kappa = 0.18) o el Kit B de ELISA (Kappa =0.15) fue ligera. La prueba de Mc Nemar (Kit A: 7.11>3.84; Kit B: 9.94>3.84) indicó que no son pruebas reemplazables. Asimismo, la concordancia entre el Kit A y el Kit B de ELISA (Kappa = $0.63)$ fue sustancial, y la prueba de Mc Nemar $(1<3.84)$ mostró que son pruebas reemplazables entre sí. 


\section{Discusión}

El estudio permitió confirmar, en base a las características de crecimiento de Map (SENASA, 2000), la presencia de la infección en cinco vacas de dos de los tres establos evaluados. El estudio no empleó técnicas bioquímicas en el cultivo del Map, dada su escasa utilidad para la correcta identificación del patógeno (Paolicchi et al., 2003). Es por esto que se empleó el PCR, el cual permitió confirmar la presencia de Map en dos animales del establo A y que, además, fueron positivas al cultivo.

La sensibilidad de PCR puede variar dependiendo del método de extracción (Odumeru et al, 2001). En este estudio se siguió el protocolo recomendado por Collins (Collins et al., 2006), donde el método de extracción de ADN fue el hervido. No obstante lisar Map por hervido no es fácil y se debe emplear un mecanismo adicional para romper la pared celular y acceder al DNA a fin de incrementar su sensibilidad (Gao et al., 2009). Asimismo, Odumeru et al. (2001) sugieren remover los componentes residuales en la preparación del ADN para incrementar la sensibilidad de PCR que amplifica la secuencia de inserción IS900. En el presente estudio, si bien se usó el hervido para la extracción de ADN, el PCR se hizo a partir de colonias que crecieron en cultivo, por lo que la sensibilidad de la prueba no debería estar afectada (Paolichi et al., 2003).

Los tres cultivos que resultaron positivos a Map y que no pudieron ser confirmados por PCR, pueden tratarse de otras micobacterias. Esto se puede ver en los resultados de la prueba tuberculínica intradérmica donde 18 animales fueron positivos y solo en dos de ellos se pudo confirmar la presencia de Map por cultivo y PCR. La prueba tuberculínica intradérmica, además de su baja sensibilidad, da reacciones cruzadas con otras micobacterias debido a que comparten antígenos comunes (Paolicchi et al., 2003). Además, en esta prueba no se usó el
PPD de Map (Jhonina) sino el PPD aviar que proviene de $M$. avium. Esto puede explicar el crecimiento de colonias compatibles a Map y negativas a PCR de muestras procedentes de animales positivos a la prueba tuberculínica intradérmica. Por otro lado, estas probables micobacterias no necesariamente serían patógenas.

La observación de heces con consistencia disminuida en algunos animales del establo A, pese a los resultados, no podría descartarse que sea debido al Map, ya que este patógeno, al ser eliminado en forma intermitente, no siempre puede ser diagnosticado por cultivo o PCR (De Juan Ferré, 2005). Para poder confirmar la posibilidad de encontrar otras especies de micobacterias que puedan causar enfermedades entéricas en los rumiantes es necesario realizar otras pruebas, tales como amplificación y secuenciación de ADN a partir de colonias de cultivo de muestras de heces. Se descarta la posibilidad de encontrar bacterias como Nocardia, por tratarse de bacterias con crecimiento rápido (Vadillo et al., 2002).

A pesar de las desventajas de la prueba tuberculínica intradérmica, esta prueba permitió detectar 18 animales positivos, donde en cinco de ellos se pudo aislar e identificar Map y en dos se pudo amplificar la secuencia de inserción IS900 por PCR. Por otro lado, no hubo casos donde se detectó Map que no hayan sido positivos a la prueba tuberculínica intradérmica, lo que resalta la utilidad diagnóstica de esta prueba. Es conocido que Map ocasiona una respuesta inmune celular inicial dada su característica de ser un agente intracelular, donde el animal permanece asintomático pero elimina el agente en forma intermitente. Es por esto, la utilidad de la prueba intradérmica que sigue siendo recomendada como prueba tamiz por los laboratorios de referencia de la OIE, dado su bajo costo y fácil manejo.

La prueba de ELISA tiene una baja sensibilidad (30 $\pm 5 \%)$ para Map (Collins et al., 2006), de allí la decisión de emplear dos kits 
en el estudio a fin de incrementar la sensibilidad y poder detectar un mayor número de animales positivos. El kit comercial A solo detectó a 7 animales y el kit B detectó a 5, pero entre los dos se detectó a 8 animales positivos (Cuadro 1). No obstante, solo en el animal positivo a los dos kits se pudo confirmar la presencia de Map, tanto por las características de crecimiento como por PCR; sin embargo, cuatro animales positivos al cultivo y uno al PCR fueron negativos a los dos kits de ELISA. De Juan Ferré (2005) y McKenna et al. (2006) mencionan que existe una relación directa entre el estado clínico del animal con la sensibilidad de la prueba ELISA, siendo de 15 a $25 \%$ en los estadios preclínicos de la enfermedad. Además, hay que tener en cuenta que la proporción de bovinos que eliminen Map por las heces en pequeñas concentraciones pueden ser subestimados, debido a que también se elimina Map durante la descontaminación de la muestra (Gao et al., 2009).

La evaluación de las dos pruebas de ELISA (Kits A y B) con la Prueba Tuberculínica Intradérmica mediante la prueba Kappa y la prueba de Mc Nemar indicó una concordancia ligera y que estas pruebas no fueron reemplazables entre sí, lo cual es un resultado esperado debido a que la prueba de ELISA detecta una respuesta inmune humoral y la prueba tuberculínica intradérmica detecta una respuesta inmune celular. Sin embargo, fue interesante encontrar que se puede optar por cualquiera de los dos kits comerciales para hacer el diagnóstico.

Por último, se debe considerar que la vía principal del proceso inmunitario en los primeros años es la respuesta inmune celular, que con el transcurso del tiempo coexiste con la respuesta inmune humoral y que, al final, predomina esta última. En este estudio, al tratarse de animales que no presentaron signos clínicos compatibles a la enfermedad de Johne y fueron positivos a las técnicas diagnósticas, se podría deducir que estarían pasando por un estadio intermedio de la infección (OIE, 2005). El estudio demostró la utilidad de las metodologías empleadas, ya que pudieron evidenciar tanto una respuesta inmune celular como humoral y la detección del agente.

\section{Conclusiones}

? Se confirma la infección por Map en bovinos lecheros en establos del departamento de Lima.

? La utilización de pruebas que evalúan la respuesta inmune celular y respuesta inmune humoral son útiles para evaluar la presencia de animales reactores a Map.

? Se encontró una concordancia ligera entre las pruebas que evalúan la respuesta inmune celular y la respuesta inmune humoral, siendo estas pruebas no reemplazables entre sí.

\section{Literatura Citada}

1. Collins DM, Hilbink F, West DM, Hosie BD, Cooke MM, Lisle GW. 1993. Investigation of Mycobacterium paratuberculosis in sheep by faecal culture, DNA characterisation and the polymerase chain reaction. Vet Rec 133: 599-600.

2. Collins MT, Gardner IA, Garry FB, Roussel AJ, Wells SJ. 2006. Consensus recommendations on diagnostic testing for the detection of paratuberculosis in cattle in the United States. JAVMA 12: 1912-1919.

3. Coussens PM. 2004. Model for immune responses to Mycobacterium avium subspecies paratuberculosis in cattle. Infect Immun 72: 3089-3096.

4. De Juan Ferré L. 2005. Paratuberculosis caprina: aportaciones a su diagnóstico, epidemiología molecular y control. Tesis doctoral. Madrid: Universidad Complutense de Madrid. 291 p.

5. Feller M, Huwiler K, Stephan R, Altpeter $E$, Shang A, Furrer $H$, Pfyffer, GE, et al. 2007. Мycobacterium avium subspecies paratuber- 
culosis and Crohn's disease: a systematic review and meta-analysis. Lancet Infect Dis 7: 607-613.

6. Gao A, Odumeru J, Raymond M, Hendrick S, Duffield T, Mutharia L. 2009. Comparison of milk culture, direct and nested polymerase chain reaction (PCR) with fecal culture based on samples from dairy herds infected with Mycobacterium avium subsp. paratuberculosis. Can J Vet Res 73: 58-64.

7. Gonzáles A, Falcón N. 1999. Análisis de datos en Medicina Veterinaria. Pub Tec. FMV $\mathrm{N}^{\circ}$ 49. LIMA: FMVUNMSM. 55 p.

8. Harris NB, Barletta RG. 2001. Mycobacterium avium subsp. paratuberculosis in Veterinary Medicine. Clin Microbiol Rev 14: 489-512.

9. Hermon-Taylor J, Bull T. 2002. Crohn's disease caused by Mycobacterium avium subspecies paratuberculosis: a public health tragedy whose resolution is long overdue. J Med Microbiol 51: 3-6.

10. Huntley JFJ, Stabel JR, Bannantine JP. 2005. Immunoreactivity of the Mycobacterium avium subsp. paratuberculosis 10-kDa Lipoprotein. BMC Microbiology 5: 3. [Internet]. Available in: http://www.biomed central.com/1471-2180/5/3

11. Jungersen G, Huda A, Hansen JJ, Lind P. 2002. Interpretation of the gamma interferon test for diagnosis of subclinical paratuberculosis in cattle. Clin Diagn Lab Immunol 9: 453-460.

12. Koo HC, Park YH, Hamilton MJ, Barrington GM, Davies CJ, Kim JB. 2004. Analysis of the immune response to Mycobacterium avium subsp. paratuberculosis in experimentally infected calves. Infect Immun 72: 68706883.

13. McKenna SLB, Barkema HW, Keefe GP, Sockett DC. 2006. Agreement between three ELISAs for Mycobacterium avium subsp. paratuberculosis in dairy cattle. Vet Microbiol 114: 285-291.
14. Motiwala AS, Amonsin A, Strother M, Manning EJB, Kapur V, Sreevatsan S. 2004. Molecular epidemiology of Mycobacterium avium subsp. paratuberculosis isolates recovered from wild animal species. J Clin Microbiol 42: 1703-1712.

15. Odumeru J, Gao A, Chen S, Raymond M, Mutharia L. 2001. Use of the bead beater for preparation of $\mathrm{Myco}$ bacterium paratuberculosis template DNA in milk. Can J Vet Res 65: 201205.

16. [OIE] World Organization for Animal Health. 2005. Old Classification of diseases notifiable to the OIE [Internet]. [2 may 2007]. Available from: http:// www.oie.int/eng/maladies/ en_OldClassification.htm\#ListeA

17. [OIE] World Organization for Animal Health. 2009. Información zoosanitaria. Información por país. Enfermedades en el tiempo. Base de datos del Sistema Mundial de Información zoosanitaria. [Internet], [26 Marzo 2009]. Disponible en: http://www.oie.int/wahis/http:// w w w.o i e.int/ w a h i s / public.php?page $=$ country_timelines\&public_country_code=PER$\&$ disease_id $=24 \&$ disease_type $=$ Terrestrial\&selected_analysis $=1 \&$ firstyear $=2005 \&$ lastyear $=2009$.

18. Pagano M, Gauvreau K. 2001. Bioestadística. $2^{\circ}$ ed. Ed. México DF: Math Learning. $584 \mathrm{p}$.

19. Paolicchi FA, Zumarraga MJ, Gioffre A, Zamorano P, Morsella C, Verna A, Cataldi A, et al. 2003. Application of different methods for the diagnosis of paratuberculosis in a dairy cattle herd in Argentina. J Vet Med 50: 20-26.

20. Schuff S. 2005. Johne's-Crohn's link gains ground. Feedstuffs 77(39): 1-3.

21. [SENASA] Servicio Nacional de Sanidad y Calidad Agroalimentaria. 2000. Manual de procedimiento técnico-diagnóstico de paratuberculosis. Laboratorio de Referencia de la Office Internacional des Epizooties (OIE) en Paratuberculosis para América del Sur, 
América Central, México y Caribe. Argentina: SENASA. $74 \mathrm{p}$.

22. Stabel JR, Bannantine JP. 2005. Development of a nested PCR method targeting a unique multicopy element, ISMap02 for detection of Mycobacterium avium subsp. paratuberculosis in fecal samples. J Clin Microbiol 43: 4744-4750.

23. Stabel JR, Bosworth TL, Kirkbride TA, Forde RL, Whitlock RH. 2004. A simple, rapid, and effective method for the extraction of Mycobacterium paratuberculosis DNA from fecal samples for polymerase chain reaction. $\mathrm{J}$ Vet Diagn Invest 16: 22-30.

24. Stewart DJ, Vaughan JA, Stiles PL, Noske PJ, Tizard MLV, Prowse SJ, et al. 2006. A long-term study in Angora goats experimentally infected with Mycobacterium avium subsp. paratuberculosis: Clinical disease, faecal culture and immunological studies. Vet Microbiol 113: 13-24.

25. Thrusfield M. 1990. Epidemiología Veterinaria. Zaragoza: Acribia. 339 p.

26. Vadillo S, Píriz S, Mateos E. 2002. Manual de microbiología veterinaria. España: McGraw-Hill/Interamericana. 853 p.

27. Waters WR, Millar JM, Palmer MV, Stabel JR, Jones DE, Koistinen KA, Steadham EM, et al. 2003. Early induction of humoral and cellular immune responses during experimental Mycobacterium avium subsp. paratuberculosis infection of calves. Infect Immun 71: 5130-5138.

28. Wren G. 1998. Testing for Johnes's. Bovine Vet, July-August: 4-8.

29. Zuñiga B. 1999. Seroprevalencia del Mycobacterium paratuberculosis en bovinos lecheros del Valle de Lima. Tesis de Medico Veterinario. Lima: Universidad Mayor de San Marcos. 37 p. 